\title{
José Gustavo Guerrero y la diplomacia salvadoreña del martinato
}

MIDORI IJIMA

Martínez reconoció asimismo al Manchukúo, el Estado títere del imperialismo japonés en Manchuria ROQUE DALTON ${ }^{1}$

\section{Introducción}

Una de las figuras salvadoreñas más homenajeadas del siglo XX es, sin lugar a duda, el Dr. José Gustavo Guerrero (1878-1958). Este jurista internacional desempeñó sucesivamente cargos tan importantes como juez, vicepresidente y presidente en la Corte Permanente de Justicia Internacional (de 1930 a 1946); tras finalizada la Segunda Guerra Mundial, nuevamente presidente, vicepresidente y juez en la actual Corte Internacional de La Haya (de 1946 hasta su muerte) ${ }^{2}$.

Su mandato como árbitro en la arena del derecho internacional a su vez, curiosamente, coincide con otro mandato peculiar en la bistoria salvadoreña: el régimen del general Maximiliano Hernández Martínez (1931-34, 1935-44).

José Gustavo Guerrero y la diplomacia salvadoreña 
Abora, ¿cuál es la co-relación entre estos dos elementos? El llamado Martinato, especialmente la década de los años treinta, se destaca por su política doméstica en torno al suceso de 1932. En cambio, poco se sabe sobre su política exterior salvo la negación del reconocimiento inmediato por parte de los Estados Unidos o algunas señales de inclinación fascistoide del propio presidente Martínez. Sin embargo, existe una cuestión crucial entre dos hombres: el reconocimiento de Manchukuo por el Gobierno salvadoreño, un insólito becho acaecido en marzo de 1934, poco después de que el régimen de Martínez obtuviera el visto bueno del flamante presidente norteamericano Franklin D. Roosevelt (1933-45 en el cargo).

\section{Manchukuo y la comunidad internacional}

A partir de la restauración imperial de Meiji en 1868, el Estado japonés opta por una "occidentalización" acelerada. Su deseo de ingresar en el club de las potencias mundiales le empuja a incurrir en una serie de guerras colonial-expansionistas. En menos de tres décadas, este imperio logra arrancarle a China parte de su territorio (la peninsula de Leaotong y la isla Taiwán); diez años más tarde, marca otra victoria ante Rusia que permite dirigir su codicia hacia el norte; en 1910 anexa la península coreana, lo cual genera un inextirpable resentimiento bacia Japón entre la población vecina.

El auto-proclamado Manchukuo, que forma parte de esta estrategia imperial, es establecido en marzo de 1932, como fruto de la intriga japonesa orquestada por el ejército Kanto, destacamento especial en Manchuria (la región nor-oriental de la actual República Popular de China). La comunidad internacional reacciona rápidamente al caso. La Sociedad o Liga de las Naciones, con sede en Ginebra, elabora un informe y condena la agresión japonesa emitiendo una nota en la que se exhorta, tanto a los Estados miembros, como a aquellos que no pertenecen a la Sociedad, a que no reconozcan ni de forma explicita, ni implícita ${ }^{3}$.

Como se deduce, el "primer" país que otorga el reconocimiento a Manchukuo es su tutor, es decir, el Imperio japonés, a través de un Convenio suscrito entre ambos en septiembre de 1932. ¿Quién siguió? Para sorpresa de todos, aparece un pequeño y lejano país llamado El Salvador. De acuerdo con varias fuentes históricas ${ }^{4}$, el 3 de 
marzo de 1934, el canciller salvadoreño Miguel Angel Araujo acusa recibo de la comunicación por parte de su homólogo Hsieb Chieb-Shih, enviada vía telegráfica desde Shinking, la capital del Estado en cuestión, en idioma inglés y fechada el $1^{\circ}$ de marzo del mismo año, que anuncia el ascenso al trono de $\mathrm{Pu}-\mathrm{Yi}$, el último emperador (1908-12) de la extinta dinastía Quin y hasta entonces el jefe de Estado de Manchukuo, abora como emperador del nuevo Imperio Manchukuo. En consecuencia, El Salvador se convierte prácticamente en el primer pais del mundo que se atreve a ignorar el llamamiento de la Sociedad de las Naciones, siendo miembro de ésta.

¿En qué consiste el móvil de este reconocimiento? Analizaremos en otra ocasión con más detenimiento la dinámica de la época que lleva a situar a El Salvador de pocos años después de la Matanza, en un escándalo internacional. Por el momento, sólo nos limitaremos a apuntar algunos hechos relevantes al respecto:

i. En realidad, desde los años anteriores El Salvador ha venido manifestando su interés poco esclarecido hacia el Oriente. Quien encarna este interés es León Sigüenza —más conocido como escritor, el primer cónsul general en Japón. Este personaje llega a Tokio en octubre del año 1927, es decir, bajo la supuesta instrucción del Gobierno de Pío Romero Bosques.

ii. Existe un documento en que la autoridad salvadoreña justifica dicho acto singular. Se trata de un folleto ${ }^{6}$, con la fecha del año 1935, atribuido a Ramón López Jiménez, subsecretario de Relaciones Exteriores entre 2 de mayo de 1931 y $1^{\circ}$ de diciembre del mismo año ${ }^{7}$, en otras palabras, hasta que el golpe de estado depusiera al presidente Arturo Araujo. Titulado "Por qué reconoció la República de El Salvador al Estado de Manchoukuo [sic.]", este folleto inclusive es traducido casi de inmediato al japonés.

iii. El sector oficial en Japón queda complacido para siempre -por lo menos hasta la década 1970- de la actitud salvadoreña y califica al país centroamericano de su "mejor amigo" tanto en América Latina como en el mundo?.

\section{Lo que mandan la moral y el derecho}

Lejos de su tierra natal, desde La Haya, el vicepresidente de la Corte Permanente de Justicia Internacional se dirige de carácter con

\section{3}

José Gustavo Guerrero y la diplomacia salvadoreña 
fidencial a San Salvador, a la mismisima persona del canciller salvadoreño. En esta carta que trae la fecha 14 de julio de 1934'10, Gustavo Guerrero se opone claramente al acto tomado por su gobierno. Vamos a recorrer su argumento (los corchetes indican muestras acotaciones):

...Nadie ignora el origen del nuevo Imperio Mandchukuo [sic.], producto de un golpe de violencia de un país fuerte [Japón] contra un Estado débil [China], que se encontraba en inferioridad evidente para hacer respetar su integridad territorial.

Antes que nada, Guerrero afirma que ba dedicado todos sus esfuerzos por adquirir para El Salvador un puesto honorable. Por tanto El Salvador, en el ámbito internacional, debe actuar de acuerdo con la moral y lo jurídico. El reconocimiento de Manchukuo contradice ese espíritu o deber moral de El Salvador.

...El Salvador, que siempre se ha elevado con legítima energía contra los atropellos de la fuerza, se encuentra por este motivo en el deber moral de no sancionar [permitir] ningún acto de esa naturaleza.

Aparte de la lógica de la moral, desde el punto de vista netamente jurídico, el acto de reconocimiento viola la regla del juego de la comunidad internacional, porque

... El Pacto de la Sociedad de las Naciones condena todo acto contrario a la integridad territorial de uno de sus miembros...

que es, en este caso concreto, China. Además, El Salvador al actuar así, desconoce

la decisión de una Asamblea extraordinaria de la Sociedad de las Naciones [celebrada en febrero de 1933], que debe ser acatada por todos los miembros de la Liga, que hayan participado o no al voto definitivo de dicha Asamblea.

Para Guerrero, todo está claro. Simple y sencillamente, el reconocimiento de Manchukuo por el Gobierno de El Salvador es un becho inadmisible. Pero su intención va más allá. Él plantea medidas para salvar el honor del país. Como hemos mencionado, siempre le importa que El Salvador, por pequeño que sea, goce de respeto y de trato, igualitario entre paises más grandes. 
...Llego ahora a la cuestión importante de saber cómo es que El Salvador puede justificar su conducta y evitar reproches morales que podrían afectar el crédito [fama] de que goza en el dominio internacional...

Su observación apunta a dos posibilidades: 1) existe un decreto de reconocimiento, publicado en el Diario Oficial ${ }^{11}$; 2) En caso contrario, la solución será levantar una excusa ante la Liga aduciendo el canje de notas entre dos cancilleres constituye únicamente el acto de la cortesía, y nunca el reconocimiento oficial. Si se diera la primera posibilidad, entonces El Salvador podría excusarse ya que no acreditó su representante ante la Asamblea extraordinaria de la Liga. Todavía habría espacio para rectificar el asunto sometiéndose de nuevo a la decisión de la Liga o de la Corte. Según sus propias expresiones, ése sería un gesto "elegante".

Por último, Guerrero trata de convencer a Miguel Ángel Araujo con pragmatismo. Sostiene que "del Mandchukuo [sic.] nada podemos esperar"; si mucho de las potencias que forman el organismo ginebrino. Obviamente el jurista sugiere que el Gobierno de El Salvador rectifique su postura para lograr, por ejemplo, algún empréstito en Europa.

\section{4. ¿Resentimiento o principio?}

Tres semanas después, el canciller Araujo, también de forma confidencial, contesta a Guerrero ${ }^{12}$. El funcionario no niega que el Manchukuo nació "si se quiere, al amparo de las armas del Japón" pero rechaza que el reconocimiento fuera un acto inconsciente. Su gobierno se tomó en cuenta "su [de Mancbukuo] funcionamiento normal durante más de dos años" al reconocerlo. En cambio, respecto a la Sociedad de las Naciones, Araujo manifiesta su crítica y decepción hacia ese organismo, dominado -según su percepción-por un pequeño grupo de naciones grandes. Además, argumenta, puesto que El Salvador no acreditó ningún delegado para la Asamblea en que se acordó no reconocer a Manchukuo, la resolución tomada en ese entonces no impone compromiso alguno ante el Gobierno de El Salvador. Inclusive para él, perjudicaría mucho más al país si retirara el reconocimiento.

En su argumentación, claramente se percibe un resentimiento hacia el organismo ginebrino ${ }^{13}$. Por ejemplo, cuestiona lo siguiente: 
i. La Sociedad no adopta ninguna actitud contra la Potencia [Japón] que se dice dio vida al nuevo Imperio [Mancbukuo].

ii. Ante la amenaza comunista, tampoco bizo nada contra las grandes naciones que traficaban ocultamente con la Rusia Soviética.

iii. Si El Salvador fuera expulsado de la liga, eso le permite suprimir del presupuesto, una partida de gastos que ningún provecho trae a la Nación.

iv. Cuando El Salvador solicitó la intervención de ese organismo [en torno a la legitimidad del presidente Martinez], sólo recibió la indiferencia.

Agrega que El Salvador no tiene intereses materiales en el Extremo Oriente y que el reconocimiento se hizo por principio, en acatamiento al Derecho Internacional.

Respecto al interrogatorio de Guerrero si el reconocimiento ha sido registrado en el Diario Oficial, el canciller Araujo explica que no existe decreto alguno ya que el asunto basta con un simple intercambio de autógrafas entre jefes de Estado, o telegramas cruzados por las cancillerías. Concluye su nota confidencial agradeciendo a Guerrero las sugerencias que se originan de su "sano patriotismo".

Parece paradójico, pero el mejor jurista del mundo no logra convencer a su compatriota canciller, y El Salvador no revocaría su reconocimiento basta enero de $1943^{14}$, trece meses después de su declaración de guerra contra Japón.

\section{Paisaje después de la batalla}

Entre los libros escritos por Gustavo Guerrero, en su mayoría en francés, $\mathrm{El}$ orden internacional, edición a cargo de la Universidad Autónoma de El Salvador (actual Universidad de El Salvador) representa una excepción. Aunque esa versión en español no tiene fecha de publicación, se presume de 1948 o 1949 gracias a algunos detalles que aparecen en el frontispicio. En su Capitulo segundo, el autor dedica unas doce páginas al "Asunto Chino-Japonés 1931". Una vez más Guerrero rechaza tajantemente la acción japonesa como agresión. Para él, "la anexión de la Manchuria" se dio "bajo la ficción de un pseudo Estado independiente", de modo que "los miembros de la Sociedad estaban obligados a negar su reconocimiento a cualquier situación crea- 
da por medios contrarios al Pacto de la Sociedad de las Naciones o al Pacto de París"'s. ¿Cómo califica entonces, a la altura de finales de la década 1940, el fulminante becho de reconocimiento ejecutado por El Salvador?

Para nuestra sorpresa, José Gustavo Guerrero, con cuyo nombre está bautizada la biblioteca de la cancillería, no dicta ni una palabra sobre el asunto. Una parte muy importante de la historia salvadoreña se oculta completamente entre sus líneas. La única explicación posible es la negligencia intencional ya que, de acuerdo con su condena al imperialismo japonés, el Dr. Guerrero debería mantener la misma opinión que quince años atrás: no está de acuerdo con el reconocimiento de Manchukuo por el Martinato. Quizás la infamia le bizo quedar callado.

Cabe mencionar también la obra posterior de otro experto del tema, ya citado Ramón López Jiménez. El conocedor de la diplomacia salvadoreña publica en 1969 su aporte más teórico: Tratado de derecho internacional público en dos tomos ${ }^{16}$. En este trabajo donde se abarcan todos los principios básicos del derecho internacional, el abogado de la independencia de Manchukuo tampoco hace ninguna referencia al acto que tomó su gobierno treinta y cinco años atrás - a menos que fuera nuestro descuido revisar sin ser exhaustivos-. Una vez más, el silencio.

¿Cómo deberíamos interpretar ese silencio? Lo más lógico de todo sería presumir lo siguiente: independientemente de la postura de cada quien sostenida durante el Martinato, ambos prefirieron no tocar el tema precisamente para no hacer ruido alguno. Es muy probable que hayan pensado asi en salvar el honor de un pequeño país llamado El Salvador.

En cambio, nos da la mayor prioridad rescatar la sincera verdad de la diplomacia salvadoreña, sobre todo cuando nos importa la dignidad de pequeños países y cuando sabemos que el Dr. Guerrero es considerado como símbolo de la política exterior salvadoreña. Como reconoce el mismo autor de la obra teórica: "El acto internacional más conocido de la República de El Salvador, en defensa del Principio de No Intervención, es la brillante actuación del doctor José Gustavo Guerrero, en la Sexta Conferencia Interamericana celebrada en 1928 en la ciudad de La Habana..." "17. Poco después de la clausura de esta Confe-

\section{7}

José Gustavo Guerrero y la diplomacia salvadoreña 
rencia, en El Salvador organizan una serie de actividades de bomenaje para Guerrero. Y entre los miembros del Comité Pro-Homenaje, llama nuestra atención el nombre del general Maximiliano Hernández Martínez ${ }^{18}$.

Para el poeta Roque Dalton, también revolucionario, la actitud del Martinato, o más concretamente la del propio Martínez, significa un apoyo cómplice al imperialismo japonés. Hoy en día, curiosamente, circula una versión totalmente desmitificadora entre ex-funcionarios del Ministerio de Relaciones Exteriores. Se cree que el "reconocimiento" fue un error cometido por algún funcionario de baja categoría quien, como si fuera de la rutina, contestó el telegrama enviado desde Manchuria sin baber consultado a sus superiores. De acuerdo con el testimonio del Dr. Alfredo Martínez Moreno, sólo la vergüenza de revelar y reconocer el "error" tan absurdo obligó al Gobierno de Martínez seguir justificando su postura ya lanzada ante todo el mundo ${ }^{19}$.

Son dos tesis completamente opuestas y no sabemos en este momento cuál tendría la razón. Falta muchísimo explorar y contextuar la documentación no sólo de parte salvadoreña, sino también de otras partes involucradas para poder llegar a la conclusión de este peculiar caso bistórico. Al igual que el silencio en que se refugiaron tanto Guerrero como López Jiménez, lo interesante es, sea cierta o no esa versión, el mismo becho de que circula hoy esa versión casi en broma entre los allegados a la cancillería ${ }^{20}$, quienes son ya de mayor edad pero están muy activos y dispuestos a asesorar a los funcionarios actuales.

En ese sentido, valdría la pena cuestionar cuál ba sido o es el eje central de la diplomacia salvadoreña y evaluar la trayectoria del Dr. José Gustavo Guerrero, factor más destacado en ese campo a lo largo de la bistoria salvadoreña. Como hemos observado rápidamente en este articulo, al tratar de su figura, bay que tener en cuenta el episodio quizás poco conocido del enfrentamiento con el Martinato.

\section{NOTAS}

1. "Martínezkampf" en Las historias probibidas del pulgarcito.

2. López Jiménez, Ramón. Tratado de derecho internacional público, San Salvador, Ministerio de Educación (Dirección de Publicación), 1969, Tomo I, 276. 
3. Sociedad de las Naciones. C.L. 117. 1933. VII. fechada 14 de junio de 1933 (El Salvador, Archivo del Ministerio de Relaciones Exteriores, año 1933, archivo 550: 348-364). En marzo de 1933, Japón se retira de la Sociedad debido a su desacuerdo.

4. Como principal fuente, Ministerio de Relaciones Exteriores (MRE), año 1934, archivo 801: 191-194.

5. MRE, año 1927 , archivo $702: 7681$.

6. Library of Congress (Washington, D.C.), F1486. L66.

7. MRE, Tratados, convenciones y acuerdos internacionales vigentes en El Salvador, Tomo I 1865-1929, San Salvador, Ministerio de Relaciones Exteriores, 1938, 14.

8. Gaiko Jiho (Revue Diplomatique), Tokio, No.735, 15 de julio de 1935, 94-115.

9. Respecto de este mito: "el mejor amigo", la autora tiene estudios preliminares en japonés. Iijima, Midori (comp., y traduc.). Kinjirareta Rekishi no Shougen (Los testimonios de las historias probibidas), Tokio, Gendai Kikakushitsu Publishers, 1996; “Aru 'Shin-nichi Koku' no Tanjou_(La cuestión de "Manchoukuo” y la política exterior salvadoreña bajo el régimen de Maximiliano Hernández Martínez)", primera parte, Guifu Daigaku Kyoyobu Kenkyu Houkoku, Guifu (Japón), Universidad de Guifu, N.`32, 1995, 59-78.

10. MRE, año 1934, archivo 801: 252-254.

11. De hecho, no está registrado ningún telegrama cruzado entre Manchukuo y El Salvador en el Diario Oficial del año 1934.

12. MRE, año 1934, archivo 801: 258-261 fechada 7 de agosto de 1934.

13. El Salvador se retirará de la Sociedad de las Naciones en agosto del año 1939. (Diario Oficial, Tomo 128, N.॰ 54, 5 de marzo de 1940, 773.)

14. Cañas Dinarte, Carlos. Diccionario de autoras y autores de El Salvador, San Salvador, CONCULTURA, 2002, 413. La autora no ha logrado comprobar esta fecha en el Diario Oficial.

15. Guerrero, José Gustavo. El orden internacional, San Salvador, Universidad Autónoma de El Salvador, s.f. [circa 1948-49], 92-103.

16. López Jiménez, R. Op.cit.

17. Ibid. Tomo I, 123.

18. Una idea, un pueblo y un hombre: El Salvador en la VI Conferencia Panamericana, San Salvador, Tipografía "La Unión", 1928, 4.

19. Entrevista de la autora al Dr. Alfredo Martínez Moreno, San Salvador, 28 de junio de 2002.

20. El Lic. Pedro Escalante Arce, funcionario de la cancillería de 1971 a 1979, también atestigua la versión similar a la del Dr. Martínez Moreno (entrevista de la autora al Lic. Pedro Escalante Arce, San Salvador, $10^{\circ}$ de julio de 2002) aunque el otro testigo vivo, Don Ramón González Montalvo, de 94 años, fundamenta el móvil del reconocimiento en la amenaza comunista que representaba el eventual triunfo del Partido Comunista de China (entrevista de la autora a Don Ramón González Montalvo, Soyapango, 10 de julio de 2002). Véase también Centuria: los bechos $y$ personajes del Siglo XX en El Salvador, San Salvador, El Diario de Hoy, 1999, 42. 